DOI: $10.33764 / 2687-041 X-2021-1-38-44$

\title{
КООРДИНИРОВАНИЕ ОБЪЕКТОВ НЕДВИЖИМОСТИ СПОСОБОМ ЭЛЕКТРОННОЙ ТАХЕОМЕТРИИ
}

\section{Евгений Ильич Аврунев}

Сибирский государственный университет геосистем и технологий, 630108, Россия, г. Новосибирск, ул. Плахотного, 10, кандидат технических наук, доцент, директор Института кадастра и природопользования, тел. (383)344-31-73, e-mail: kadastr204@yandex.ru

\section{Александра Андреевна Пинигина}

Сибирский государственный университет геосистем и технологий, 630108, Россия, г. Новосибирск, ул. Плахотного, 10, обучающийся, тел. (983)129-11-44, e-mail: 21807А@mail.ru

В статье рассматривается проблематика современного состояния вопроса по координатному обеспечению землеустроительной и кадастровой деятельности. На примере кадастрового квартала города Новосибирск рассматривается координирование объектов недвижимости способом электронной тахеометрии с прямой и без прямой оптической видимости между исходными пунктами. В результате сравнения полученных точностных параметров запроектированного геодезического построения с нормативными значениями делается заключение о целесообразности применения данного способа.

Ключевые слова: объекты недвижимости, координирование, электронная тахеометрия, государственный кадастровый учет, наземные измерительные технологии, характерные точки

\section{COORDINATION OF REAL ESTATE OBJECTS USING ELECTRONIC TOTAL STATIONS}

\section{Evgeny I. Avrunev}

Siberian State University of Geosystems and Technologies, 10, Plakhotnogo St., Novosibirsk, 630108, Russia, Ph. D., Director of the Institute of Cadastre and Environmental Management, phone: (383)344-31-73, e-mail: kadastr204@yandex.ru

\section{Alexandera A. Pinigina}

Siberian State University of Geosystems and Technologies, 10, Plakhotnogo St., Novosibirsk, 630108, Russia, Student, phone: (983)129-11-44, e-mail: 21807A@mail.ru

The article deals with the problems of the current state of coordination of land management and cadastral activities. The method of electronic total stations with and without direct optical visibility between the points of origin is considered on the example of the cadastral quarter in the city of Novosibirsk. The obtained values are compared with the existing requirements. It is concluded that it is advisable to use this method.

Keywords: real estate objects, coordination, electronic total station, state cadastral registration, ground-based measurement technologies, characteristic points

Важнейшим компонентом выполнения кадастровых работ является координирование объектов местности, которые необходимо поставить на государственный кадастровый учет (ГКУ). Для выполнения данной технологической процеду- 
ры кадастровому инженеру необходимо подготовить межевой или технический план, основным разделом которого является информация о местоположении объекта недвижимости, в отношении которого проводятся кадастровые работы.

В настоящее время существует достаточно большое количество способов координирования, детально рассмотренных во многих научно-технических статьях и монографиях [1-5]. Однако способу электронной тахеометрии, основанному на применении, традиционных наземных измерительных технологий, по нашему мнению, не уделено достаточного внимания.

Достоинством данного способа координирования является возможность одновременного координирования характерных точек, закрепляющих на местности контур объекта недвижимости, и построение геодезического съемочного обоснования. Наиболее технологичным этот способ становится при применении безотражательных электронных тахеометров, позволяющих при минимальной трудоемкости и с высокой точностью координировать углы объектов капитального строительства.

Принцип построения на местности хода электронной тахеометрии представлен на рисунке, где на кадастровый квартал 54:35:073100 города Новосибирск выполнен проект такого способа координирования.

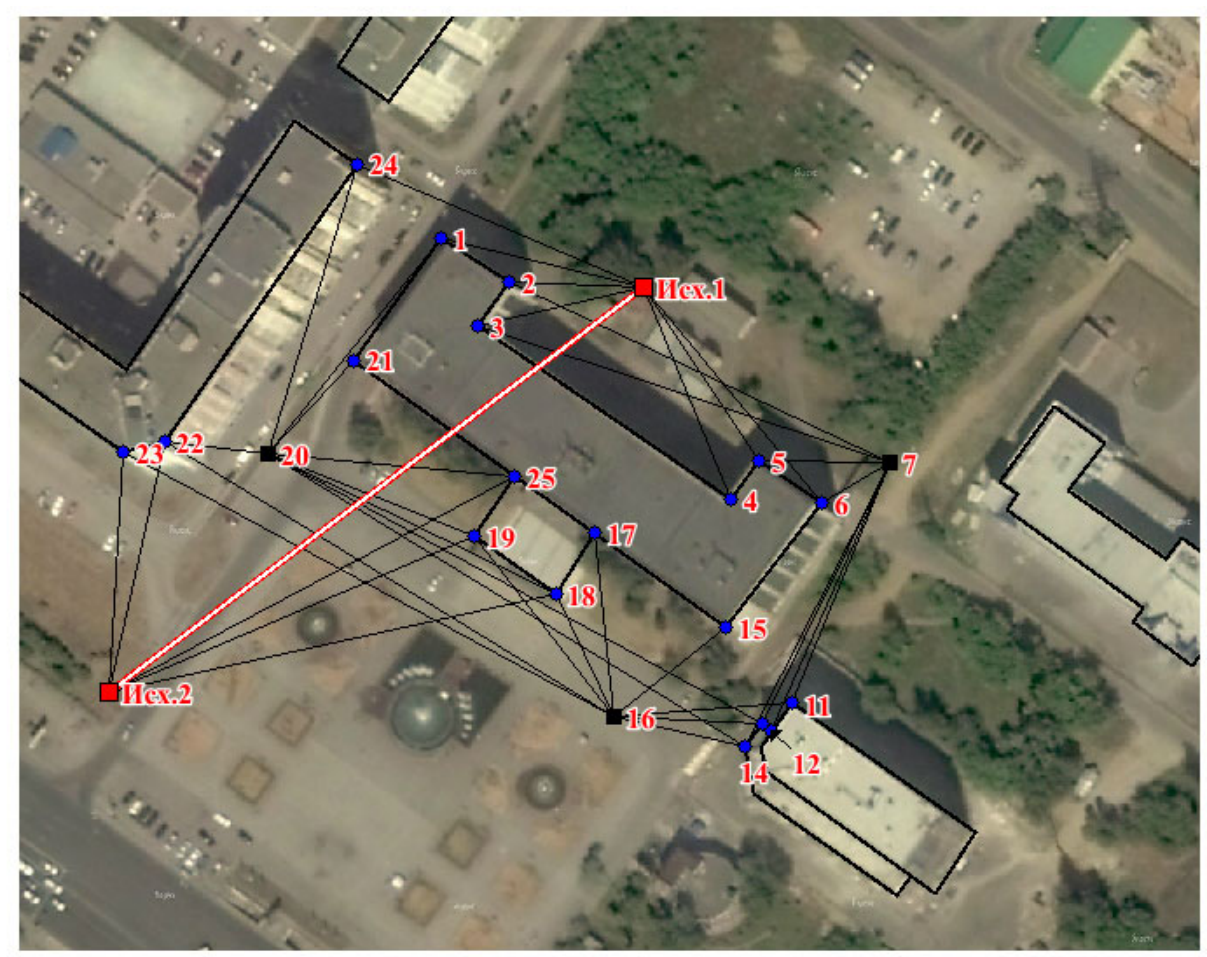

УСЛОВНЫЕ ОБОЗНАЧЕНИЯ:

- Исходные пункты;

- Связующие точки теодолитного хода, являющиеся одновременно

- характерными точками местности, которые необходимо закоординировать;

- Промежуточные точки теодолитного хода, которые

являются местами установки электронного тахеометра;

Измеряемые элементы электронной тахеометрии (углы и длины линий);

Прямая оптическая видимость между исходными пунктами.

Схема хода электронной тахеометрии 
Методика построения на местности хода электронной тахеометрии и математической обработки результатов измерений заключается в следующем:

1. По выполненным измерениям с исходного пункта 1 выполняется вычисление координат промежуточных точек $\left(\mathrm{X}_{24}, \mathrm{Y}_{24}, \mathrm{X}_{1}, \mathrm{Y}_{1}, \mathrm{X}_{2}, \mathrm{Y}_{2}, \mathrm{X}_{3}\right.$, $\mathrm{Y}_{3}, \mathrm{X}_{4}, \mathrm{Y}_{4}, \mathrm{X}_{5}, \mathrm{Y}_{5}, \mathrm{X}_{6}, \mathrm{Y}_{6}$, ) с использованием алгоритма прямой геодезической задачи;

2. Со связующей точки 7 (месте установки электронного тахеометра) выполнение измерений на промежуточные точки $(2,3,4,5,6)$ и с использованием алгоритма уравнивания обратной линейно-угловой засечки вычисление координат этой связующей точки $\left(\mathrm{X}_{7}, \mathrm{Y}_{7}\right)$;

3. Выполнение измерений со связующей точки 7 на новые промежуточные характерные точки $(11,12,13,14)$ и вычисление их координат;

4. Аналогично выполняется все запроектированные измерения до того момента, когда тахеометр устанавливается на исходный пункт 2;

5. Контроль точности выполненных измерений заключается в сравнении координат исходного пункта 2 , которые приведены в каталоге, и его значений, полученных по ходу электронной тахеометрии.

Исследование точности параметров хода электронной тахеометрии выполнялось в строгом соответствии с методом наименьших квадратов в специализированном программном обеспечении, разработанном на кафедре кадастра и ТП [2].

Результаты оценки точности положения пунктов относительно ближайшего исходного приведены в табл. 1.

Таблица 1

Результаты оценки точности параметров хода электронной тахеометрии

\begin{tabular}{|c|c|c|c|c|c|c|c|}
\hline Пункт & \multicolumn{1}{l|}{$\mathrm{m}_{\mathrm{X}}$} & \multicolumn{1}{l|}{$\mathrm{m}_{\mathrm{Y}}$} & \multicolumn{1}{l|}{$\mathrm{m}$} & Пункт & $\mathrm{m}_{\mathrm{X}}$ & $\mathrm{m}_{\mathrm{Y}}$ & $\mathrm{m}$ \\
\hline 1 & 0,2 & 0,2 & 0,3 & 15 & 0,4 & 0,4 & 0,5 \\
\hline 2 & 0,1 & 0,3 & 0,3 & $\underline{16}$ & 0,2 & 0,2 & 0,3 \\
\hline 3 & 0,2 & 0,3 & 0,3 & 17 & 0,4 & 0,3 & 0,5 \\
\hline 4 & 0,4 & 0,3 & 0,5 & 18 & 0,2 & 0,2 & 0,3 \\
\hline 5 & 0,2 & 0,2 & 0,3 & 19 & 0,2 & 0,2 & 0,3 \\
\hline 6 & 0,2 & 0,2 & 0,3 & $\underline{20}$ & 0,2 & 0,3 & 0,3 \\
\hline$\underline{7}$ & 0,3 & 0,2 & 0,4 & 21 & 0,4 & 0,3 & 0,5 \\
\hline 11 & 0,3 & 0,3 & 0,4 & 22 & 0,2 & 0,2 & 0,3 \\
\hline 12 & 0,3 & 0,3 & 0,4 & 23 & 0,3 & 0,2 & 0,4 \\
\hline 13 & 0,3 & 0,3 & 0,4 & 24 & 0,3 & 0,3 & 0,4 \\
\hline 14 & 0,3 & 0,3 & 0,4 & 25 & 0,2 & 0,3 & 0,4 \\
\hline
\end{tabular}

Примечание: результаты оценки точности параметров приведены в см.

Результаты оценки точности взаимного положения характерных точек $\left(\mathrm{m}_{\mathrm{i}-\mathrm{j}}\right)$, дирекционных углов $\left(\mathrm{m}_{\alpha}\right)$ и длин линий $\left(\mathrm{m}_{\mathrm{s}}\right)$ приведены в табл. 2. 
Таблиия 2

Результаты оценки точности параметров хода электронной тахеометрии

\begin{tabular}{|c|c|c|c|c|}
\hline $\begin{array}{c}\text { Пункт } \\
\text { стояния }\end{array}$ & $\begin{array}{c}\text { Пункт } \\
\text { наблюдения }\end{array}$ & $\mathrm{m}_{\alpha}$ (сек) & $\mathrm{m}_{\mathrm{S}}(\mathrm{cm})$ & $\mathrm{m}_{\mathrm{i}-\mathrm{j}}(\mathrm{cm})$ \\
\hline \multirow[t]{7}{*}{ Исх. 1} & 24 & 8 & 0,28 & 0,42 \\
\hline & 1 & 8 & 0,25 & 0,32 \\
\hline & 2 & 8 & 0,31 & 0,34 \\
\hline & 3 & 8 & 0,30 & 0,34 \\
\hline & 4 & 8 & 0,40 & 0,45 \\
\hline & 5 & 6 & 0,24 & 0,29 \\
\hline & 6 & 5 & 0,23 & 0,29 \\
\hline \multirow[t]{8}{*}{7} & 11 & 11 & 0,24 & 0,42 \\
\hline & 12 & 10 & 0,21 & 0,41 \\
\hline & 13 & 9 & 0,20 & 0,38 \\
\hline & 14 & 8 & 0,21 & 0,39 \\
\hline & 6 & 9 & 0,23 & 0,24 \\
\hline & 5 & 8 & 0,25 & 0,29 \\
\hline & 3 & 6 & 0,27 & 0,45 \\
\hline & 2 & 6 & 0,29 & 0,43 \\
\hline \multirow[t]{11}{*}{16} & 15 & 12 & 0,40 & 0,45 \\
\hline & 11 & 11 & 0,25 & 0,34 \\
\hline & 12 & 9 & 0,22 & 0,29 \\
\hline & 13 & 8 & 0,20 & 0,25 \\
\hline & 14 & 7 & 0,22 & 0,25 \\
\hline & 23 & 5 & 0,25 & 0,43 \\
\hline & 22 & 4 & 0,21 & 0,36 \\
\hline & 19 & 7 & 0,21 & 0,30 \\
\hline & 18 & 9 & 0,21 & 0,26 \\
\hline & 17 & 11 & 0,40 & 0,47 \\
\hline & 1 & 5 & 0,22 & 0,41 \\
\hline \multirow[t]{11}{*}{20} & 24 & 8 & 0,28 & 0,40 \\
\hline & 1 & 7 & 0,20 & 0,31 \\
\hline & 21 & 7 & 0,40 & 0,41 \\
\hline & 25 & 7 & 0,28 & 0,35 \\
\hline & 19 & 6 & 0,21 & 0,28 \\
\hline & 18 & 5 & 0,21 & 0,30 \\
\hline & 13 & 4 & 0,22 & 0,38 \\
\hline & 14 & 4 & 0,23 & 0,39 \\
\hline & 22 & 6 & 0,20 & 0,22 \\
\hline & Исх.2 & 5 & 0,18 & 0,26 \\
\hline & 1 & 7 & 0,20 & 0,3 \\
\hline \multirow[t]{6}{*}{ Исх.2 } & 23 & 8 & 0,30 & 0,39 \\
\hline & 22 & 6 & 0,19 & 0,27 \\
\hline & 25 & 5 & 0,27 & 0,38 \\
\hline & 19 & 5 & 0,18 & 0,29 \\
\hline & 18 & 4 & 0,19 & 0,29 \\
\hline & & 7 & 0,25 & \\
\hline
\end{tabular}


На основании приведенных результатов можно сделать следующие выводы:

- в ходе электронной тахеометрии точки установки электронного тахеометра определяются практически с одной точностью и составляют $\mathrm{m}=0,3 \mathrm{~cm}$;

- наиболее грубо в ходе электронной тахеометрии определяется характерная точка ОКСа, 15 средняя квадратическая ошибка которой составляет $\mathrm{m}=0,5 \mathrm{~cm}$;

- наибольшая СКО взаимного положения характерных точек характерная для линии $17-11$ и составляет $0,5 \mathrm{~cm}$;

- при использовании электронного тахеометра с точностными характеристиками $\mathrm{m}_{\beta}=6$ " и $\mathrm{m}_{\mathrm{L}}=0,4 \mathrm{~cm}$ CКО параметров хода примерно составляют точность линейных измерений используемого технологического измерительного средства (табл. 3 и 4).

Оптимальным вариантом построения хода электронной тахеометрии является ситуация, когда между исходными пунктами отсутствует прямая оптическая видимость, в связи с чем нет возможности выполнить измерение примычных углов. В этом случае схема построения хода электронной тахеометрии будет выглядеть следующим образом (рис. 2).

Таблий 3

Результаты оценки точности параметров хода электронной тахеометрии с координатной привязкой

\begin{tabular}{|c|c|c|c|c|c|c|c|}
\hline Пункт & \multicolumn{1}{l|}{$\mathrm{m}_{\mathrm{X}}$} & \multicolumn{1}{l|}{$\mathrm{m}_{\mathrm{Y}}$} & $\mathrm{m}$ & Пункт & $\mathrm{m}_{\mathrm{X}}$ & $\mathrm{m}_{\mathrm{Y}}$ & $\mathrm{m}$ \\
\hline 1 & 0,2 & 0,3 & 0,3 & 15 & 0,4 & 0,4 & 0,6 \\
\hline 2 & 0,1 & 0,3 & 0,3 & $\underline{16}$ & 0,3 & 0,2 & 0,3 \\
\hline 3 & 0,2 & 0,3 & 0,4 & 17 & 0,5 & 0,3 & 0,5 \\
\hline 4 & 0,4 & 0,3 & 0,5 & 18 & 0,3 & 0,2 & 0,3 \\
\hline 5 & 0,2 & 0,3 & 0,3 & 19 & 0,2 & 0,2 & 0,3 \\
\hline 6 & 0,3 & 0,3 & 0,4 & $\underline{20}$ & 0,2 & 0,2 & 0,3 \\
\hline$\underline{7}$ & 0,3 & 0,3 & 0,4 & 21 & 0,4 & 0,3 & 0,5 \\
\hline 11 & 0,3 & 0,3 & 0,5 & 22 & 0,2 & 0,2 & 0,3 \\
\hline 12 & 0,3 & 0,3 & 0,4 & 23 & 0,3 & 0,2 & 0,4 \\
\hline 13 & 0,3 & 0,3 & 0,4 & 24 & 0,3 & 0,3 & 0,4 \\
\hline 14 & 0,3 & 0,3 & 0,4 & 25 & 0,2 & 0,3 & 0,4 \\
\hline
\end{tabular}

Примечание: результаты оценки точности приведены в см.

Таблица 4

Результаты оценки точности параметров хода электронной тахеометрии

\begin{tabular}{|c|c|c|c|c|}
\hline $\begin{array}{l}\text { Пункт } \\
\text { стояния }\end{array}$ & $\begin{array}{l}\text { Пункт } \\
\text { наблюдения }\end{array}$ & $\mathrm{m}_{\alpha}$ (сек) & $\mathrm{m}_{\mathrm{S}}(\mathrm{cm})$ & $\mathrm{m}_{\mathrm{i}-\mathrm{j}}$ (см) \\
\hline \multirow{2}{*}{ Исх. 1 } & 24 & 8 & 0,28 & 0,43 \\
\cline { 2 - 5 } & 1 & 8 & 0,26 & 0,33 \\
\hline & 2 & 8 & 0,31 & 0,34 \\
\hline & 3 & 9 & 0,30 & 0,35 \\
\hline & 4 & 9 & 0,40 & 0,47 \\
\hline
\end{tabular}


Окончание табл. 4

\begin{tabular}{|c|c|c|c|c|}
\hline $\begin{array}{l}\text { Пункт } \\
\text { стояния }\end{array}$ & \begin{tabular}{|l} 
Пункт \\
наблюдения
\end{tabular} & $\mathrm{m}_{\alpha}$ (сек) & $\mathrm{m}_{\mathrm{S}}(\mathrm{cm})$ & $m_{i-j}(c M)$ \\
\hline & 6 & 10 & 0,24 & 0,42 \\
\hline \multirow[t]{8}{*}{7} & 11 & 11 & 0,24 & 0,43 \\
\hline & 12 & 10 & 0,21 & 0,42 \\
\hline & 13 & 9 & 0,21 & 0,39 \\
\hline & 14 & 8 & 0,22 & 0,40 \\
\hline & 6 & 9 & 0,27 & 0,29 \\
\hline & 5 & 8 & 0,26 & 0,30 \\
\hline & 3 & 7 & 0,28 & 0,49 \\
\hline & 2 & 7 & 0,29 & 0,47 \\
\hline \multirow[t]{11}{*}{16} & 15 & 12 & 0,40 & 0,46 \\
\hline & 11 & 11 & 0,25 & 0,35 \\
\hline & 12 & 10 & 0,22 & 0,29 \\
\hline & 13 & 8 & 0,20 & 0,26 \\
\hline & 14 & 7 & 0,22 & 0,25 \\
\hline & 23 & 5 & 0,25 & 0,45 \\
\hline & 22 & 5 & 0,21 & 0,38 \\
\hline & 19 & 7 & 0,22 & 0,30 \\
\hline & 18 & 10 & 0,22 & 0,28 \\
\hline & 17 & 11 & 0,40 & 0,48 \\
\hline & 1 & 6 & 0,23 & 0,43 \\
\hline \multirow[t]{11}{*}{20} & 24 & 8 & 0,28 & 0,40 \\
\hline & 1 & 7 & 0,20 & 0,31 \\
\hline & 21 & 7 & 0,40 & 0,42 \\
\hline & 25 & 7 & 0,28 & 0,36 \\
\hline & 19 & 7 & 0,21 & 0,28 \\
\hline & 18 & 6 & 0,22 & 0,33 \\
\hline & 13 & 5 & 0,22 & 0,41 \\
\hline & 14 & 5 & 0,23 & 0,41 \\
\hline & 22 & 6 & 0,20 & 0,22 \\
\hline & Исх.2 & 6 & 0,18 & 0,27 \\
\hline & 1 & 7 & 0,20 & 0,21 \\
\hline \multirow[t]{6}{*}{ Исх. 2} & 23 & 8 & 0,30 & 0,39 \\
\hline & 22 & 6 & 0,19 & 0,28 \\
\hline & 25 & 5 & 0,27 & 0,39 \\
\hline & 19 & 5 & 0,18 & 0,31 \\
\hline & 18 & 5 & 0,20 & 0,34 \\
\hline & & 7 & 0,26 & \\
\hline
\end{tabular}

На основании приведенных результатов можно сделать следующие выводы:

- в ходе электронной тахеометрии с координатной привязкой точки установки электронного тахеометра определяются практически с такой же точностью, что и в первоначальном варианте и составляют $\mathrm{m}=0,3 \mathrm{~cm}$;

- наиболее грубо в ходе с координатной привязкой определяется характерная точка $15 \mathrm{CKO}$ которой составила $\mathrm{m}=0,6 \mathrm{~cm}$ (понижение точности составило не более $15 \%$ ); 
- наибольшая СКО взаимного положения характерных точек характерная для линии $17-11$ и составила 0,5 см (понижение точности составило также не более $15 \%$ );

- при использовании электронного тахеометра с точностными характеристиками $\mathrm{m}_{\beta}=6$ " и $\mathrm{m}_{\mathrm{L}}=0,4 \mathrm{~cm}$ CКО параметров хода примерно определяются точностью линейных измерений используемого технологического измерительного средства, при этом понижение точности определяемых параметров в случае координатной привязки составляет менее $15 \%$;

- полученные значения точностных параметров соответствуют предлагаемым нормативным требованиям при координировании объектов недвижимости в территориальном образовании [6] и, следовательно, этот способ может быть рекомендован кадастровым инженерам для выполнения геодезических работ при осуществлении кадастровой деятельности.

\section{БИБЛИОГРАФИЧЕСКИЙ СПИСОК}

1. Аврунев, Е. И. Проблемы кадастровой деятельности [Текст] / Е. И. Аврунев, А. И. Каленицкий, В. Н. Клюшниченко // Известия вузов. Геодезия и аэрофотосъемка. - 2015. № 5/C.- C. 99-103.

2. Аврунев, Е. И. Геодезическое обеспечение государственного кадастра недвижимости [Текст] / Е. И. Аврунев. - Новосибирск : СГГА, 2010.

3. Аврунѐв Е. И., Метелева М. В. О совершенствовании системы координатного обеспечения государственного кадастра недвижимости // Вестник СГГА. - 2014. - Вып. 1 (25). C. 60-66.

4. Карпик, А. П. Электронное геопространство - сущность и концептуальные основы [Текст] / А. П. Карпик, Д. В. Лисицкий // Геодезия и картография. -2009. - № 5. - С. 41-44.

5. Карпик, А. П. Сущность геоинформационного пространства территорий как единой основы развития государственного кадастра недвижимости [Текст] / А. П. Карпик, В. С. Хорошилов // Изв. вузов. Геодезия и аэрофотосъемка. - 2012. - № 1. - С. 134-136.

6. Метелева М.В. Разработка и исследование методики координатного обеспечения кадастровой деятельности в территориальных образованиях. [Электронный ресурс]: текст автореферата - https://www.rsl.ru.

(C) Е. И. Аврунев, А. А. Пинигина, 2021 